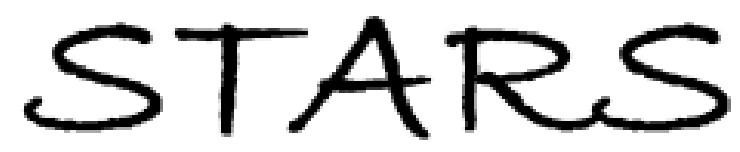

University of Central Florida

STARS

$1-1-2004$

\title{
Large electron correlation effects in the nondipole asymmetry parameters near photoionization thresholds
}

Hari P. Saha

University of Central Florida

Find similar works at: https://stars.library.ucf.edu/facultybib2000

University of Central Florida Libraries http://library.ucf.edu

This Article is brought to you for free and open access by the Faculty Bibliography at STARS. It has been accepted for inclusion in Faculty Bibliography 2000 s by an authorized administrator of STARS. For more information, please contact STARS@ucf.edu.

\section{Recommended Citation}

Saha, Hari P., "Large electron correlation effects in the nondipole asymmetry parameters near photoionization thresholds" (2004). Faculty Bibliography 2000 s. 4762.

https://stars.library.ucf.edu/facultybib2000/4762

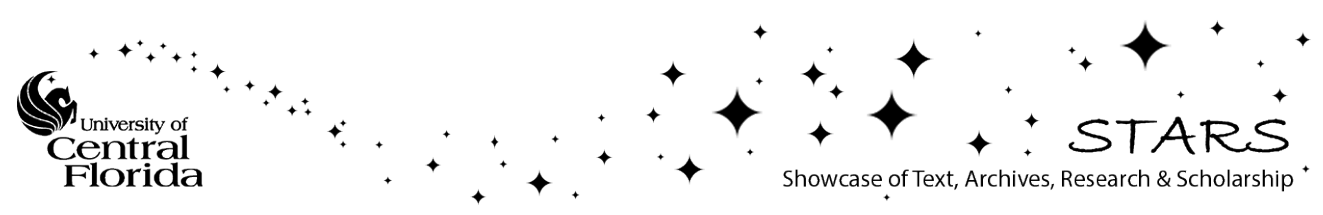




\title{
Large electron correlation effects in the nondipole asymmetry parameters near photoionization thresholds
}

\author{
Hari P. Saha* \\ Physics Department, University of Central Florida, Orlando, Florida 32816, USA
}

(Received 5 November 2003; published 25 February 2004)

\begin{abstract}
The nondipole corrections to the dipole approximation for the angular distribution of photoelectrons are calculated for Ar $3 s$ subshell in the Hartree-Fock (HF) approximation for photoelectron energies ranging from threshold to $2 \mathrm{keV}$. The effects of electron correlation which are very important have been taken into account using the multiconfiguration HF approximation. It is found that near the threshold from 0 to $50 \mathrm{eV}$ photoelectron energy, the nondipole parameters show oscillatory structure due to large electron correlation effects. The results are compared with existing theoretical and experimental data.
\end{abstract}

DOI: $10.1103 /$ PhysRevA.69.022712

PACS number(s): 32.80.Fb, 31.25.Eb

\section{INTRODUCTION}

The differential cross section of photoelectrons ejected due to atomic photoionization is an important source of information about the atomic structure and the dynamics of the process. These cross sections are very sensitive to the quality of the wave functions involved in the process. Nowadays with the advent of new synchrotron light sources around the world, experimentalists can study detailed information on the photoionization process. Experiments on measurements of the angular distribution of photoelectrons ejected due to atomic photoionization provide data not only on the relevant transition amplitudes but also on their relative phases. This data also supplies information on the initial and final states of the target as well as dynamic information on the interaction of photoelectrons with the resulting ion. The extraction of this information by a theoretical calculation is a good test of the theory.

Recently there has been a growing interest both theoretically and experimentally to investigate the effects of nondipole corrections to the angular distribution of photoelectrons [1-12]. Generally it is believed that at low photon energy the dipole approximation is sufficient to obtain useful information about the angular distribution of photoelectrons. The contributions of higher-order multipoles are expected to be very small at low photon energies. But recent theoretical and experimental investigations showed that higher-order multipoles have considerable effect on the angular distribution of photoelectrons even at low photon energies.

Theoretical studies of nondipole effects in photoionization of multielectron atoms have been performed by Amusia et al. [13] many years ago. Since then, there have been many investigations of these effects. Bechler and Pratt [14] carried out relativistic calculations of the dipole-quadrupole interference corrections to the photoelectron angular distribution for $1 s, 2 s$, and $2 p$ subshells of atoms with nuclear charges $Z$ ranging from 6 to 40 using Coulomb-field and screened Coulomb-field approximations. Scofield [15] made detailed numerical calculations for $\mathrm{Ne}$-like $\mathrm{Ba}$ and $\mathrm{He}$-like $\mathrm{Ni}$ in the

*Electronic address: hps1@physics.ucf.edu relativistic independent particle approximation (IPA) using a Dirac-Slater central potential. Cooper [1] presented extensive nonrelativistic numerical results for the nondipole asymmetry parameters for inner subshells of all noble gas atoms from $\mathrm{He}$ to $\mathrm{Xe}$ for electron energies from $100 \mathrm{eV}$ to $5 \mathrm{keV}$ using a central-field model based on the Herman-Skillman potential. Recently Dias et al. [2] reported a breakdown of the IPA in the dipole $2 p$ photoionization of $\mathrm{Ne}$ far above threshold (200-1400 eV), which indicated the need for verification of the same phenomena in the nondipole parameters. In response to this need Johnson et al. [3] extended both the relativistic random-phase approximation and the nonrelativistic random-phase approximation with exchange beyond the dipole approximation to investigate the nondipole parameters in the $\mathrm{keV}$ region and applied these methods to the $\mathrm{Ne} 2 p$ photoionization to interpret the experimental data. Later Derevianko et al. [4] presented results from a theoretical study of first- and second-order corrections to the dipole approximation in photoionization of rare gas atoms. Derevianko et al. [5] also investigated the second-order nondipole effects in the angular distribution of neon valence photoelectrons in the $100-1200 \mathrm{eV}$ photon energy range. Very recently Johnson and Cheng [6] reported strong nondipole effects in low-energy photoionization of the $5 s$ and $5 p$ subshells of xenon for photon energies below $200 \mathrm{eV}$. Recently another investigation was made by Trzhaskovskaya et al. [7] who calculated parameters of the angular distribution of photoelectrons for all subshells of atoms with $1 \leqslant Z \geqslant 54$ within the quadrupole approximation with the central Dirac-Fock-Slater potential.

Most of the theoretical investigations of the nondipole effects are confined to photon energies above $100 \mathrm{eV}$. Recently Amusia et al. [8,9] investigated in detail the nondipole effects in the angular distribution of photoelectrons near the atomic photoionization thresholds using one particle HartreeFock (HF) approximation without electron correlation and random-phase approximation with exchange (RPAE) with electron correlation. They carried out calculations for the $s$ and $p$ subshells of noble gas atoms for the photoelectron energy range from the photoionization threshold to $1.6 \mathrm{keV}$. They found oscillations in the nondipole parameters as a function of photoelectron energy near the photoionization 
threshold. This oscillatory behavior is very sensitive to electron correlations.

On the experimental side, there are two early experiments which show deviations from the dipole approximation at energies below $5 \mathrm{keV}[16,17]$. Recently experiments on the measurement of photoionization of closed shell atoms for photon energies in the range $100-5000 \mathrm{eV}$ demonstrated the breakdown of the dipole approximation. Krassig et al. [10] measured the nondipole asynmmetry parameter for $\mathrm{Ar} 1 \mathrm{~s}$ photoelectron angular distribution over $30-2000 \mathrm{eV}$ photoelectron range. Later this group [11] extended their measurements of nondipole parameters in argon $1 s$, krypton $2 s$ and krypton $2 p$ subshells within $2-3 \mathrm{keV}$ of their respective thresholds. Hemmers et al. [12] measured the nondipole parameters of the valence shell $\mathrm{Ne} 2 s$ and $2 p$ photoionization for the photon energy range $250-1000 \mathrm{eV}$.

However, most of the experimental measurements were carried out at relatively high photon energies. Currently available synchrotron radiation facilities provide the experimentalists with an intense, tunable, and highly polarized photon beam. Experimentalists will be able to measure nondipole parameter at much more interesting low photon energies where electron correlation effects are large and very important. Theoretical predictions about the effects of electron correlation on the nondipole parameters in the low photoelectron energies have already been made by Amusia $\mathrm{et} \mathrm{al}$. using the RPAE method [13]. These theoretical and experimental developments on the determination of effects of higher-order multipoles to the angular distribution of photoelectrons inspired us to look into this problem very carefully and understand the effects of electron correlation to the nondipole asymmetry parameters at low photon energies. This needed extension of the multiconfiguration Hartree-Fock (MCHF) method [18] beyond the dipole approximation to study in detail the electric dipole-quadrupole interference effects from threshold to the $\mathrm{keV}$ photon energy region.

In this paper, as a test case, we report calculations of the nondipole parameters for the $\operatorname{Ar} 3 s$ subshell photoionization from threshold to $2 \mathrm{keV}$. We first perform calculations in the HF approximation. To determine the effect of electron correlation we use the extended MCHF method. The MCHF approximation, which is capable of taking into account both electron correlation and polarization effects very accurately completely $a b$ initio, has proved to be very useful in describing angular distribution asymmetry parameter $\beta$ in the dipole approximation. Although at this moment experimental measurements of nondipole parameters are made for $\operatorname{Ar} 1 s$, this investigation will stress the need for further experimental measurements for $\mathrm{Ar} 3 s$, particularly at photon energies from threshold to $\mathrm{keV}$ region.

\section{THEORY}

The photoionization matrix element is given by

$$
\mathcal{M}_{i f}=\left\langle f\left|(\mathbf{e} \cdot \mathbf{p}) e^{i \mathbf{k} \cdot \mathbf{r}}\right| i\right\rangle,
$$

where $|i\rangle$ and $|f\rangle$ are the initial and final states of the atom respectively. $\mathbf{k}$ and $\mathbf{e}$ are photon momentum and polarization

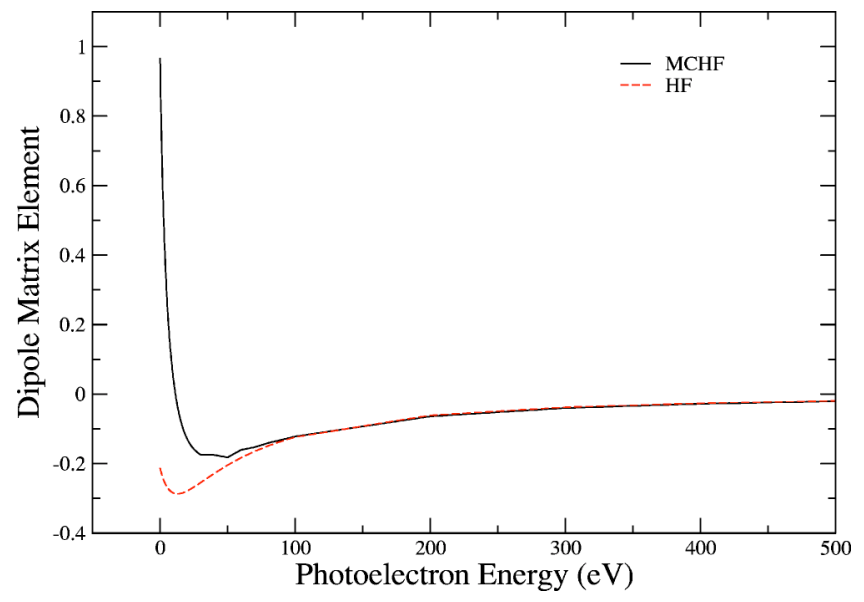

FIG. 1. (Color online) Dipole matrix elements in the MCHF and $\mathrm{HF}$ approximations as a function of photoelectron energy.

vectors, $\mathbf{r}$ and $\mathbf{p}$ are the coordinate and the momentum of the electron. Generally at low photon energies, the dipole approximation $(E 1)$ is valid. In this case $e^{i \mathbf{k} \cdot \mathbf{r}}$ is approximated by 1 . For high photon energies, the dipole approximation is no longer valid. The first-order correction to the nondipole effect is obtained by retaining up to the second term in the expansion of the exponent

$$
\mathcal{D}_{i f}=\langle f|(\mathbf{e} \cdot \mathbf{p})(1+i \mathbf{k} \cdot \mathbf{r})| i\rangle .
$$

The second term represents the amplitude of the magnetic dipole $(M 1)$ and electric quadrupole (E2) transitions. The interference of the $E 1, M 1$, and $E 2$ amplitudes leads to the general form of the angular distribution of photoelectrons. In the case considered, the interference between $E 1$ and $E 2$ amplitudes between the initial atomic state to the final continuum state describes the nondipole corrections to the differential cross section for photoionization.

For linearly polarized light, the expression for the nondipole angular distribution parameters is given by

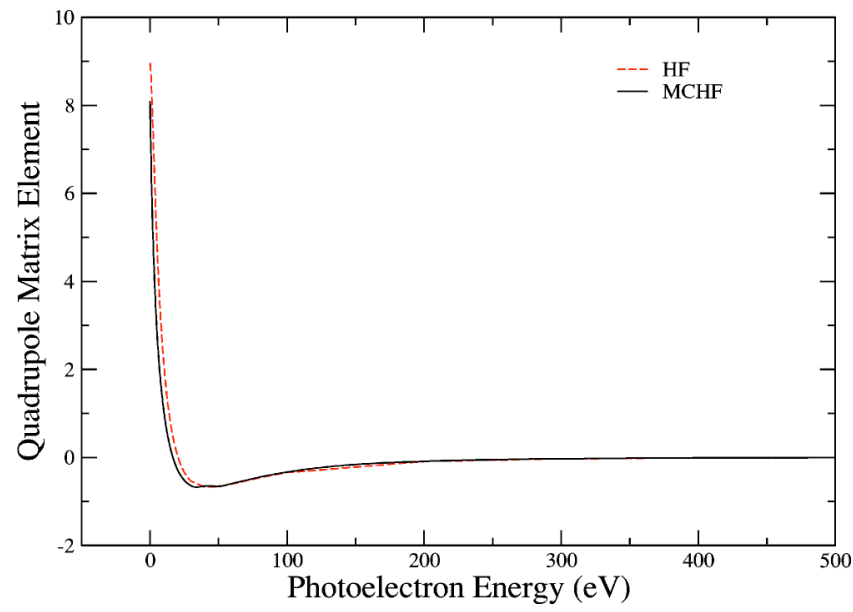

FIG. 2. (Color online) Quadrupole matrix elements in the $\mathrm{MCHF}$ and HF approximations as a function of photoelectron energy. 


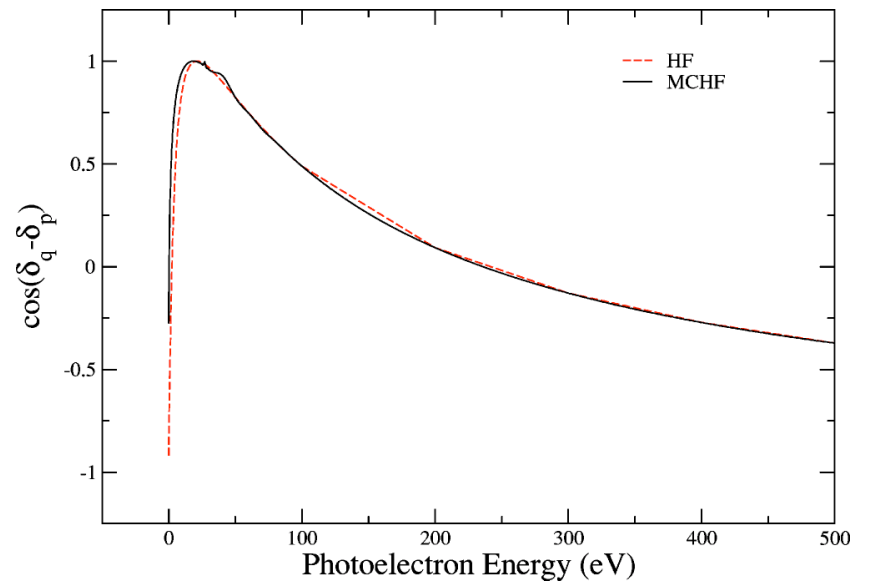

FIG. 3. (Color online) Cosine of $\left(\delta_{q}-\delta_{p}\right)$ in the MCHF and HF approximations as a function of photoelectron energy.

$$
\begin{aligned}
\frac{d \sigma_{n l}(\omega)}{d \theta}= & \frac{\sigma_{n l}(\omega)}{4 \pi}\left\{1+\beta(\omega) P_{2}(\cos \theta)\right. \\
& \left.+\left[\delta(\omega)+\gamma(\omega) \cos ^{2} \theta\right] \sin \theta \cos \phi\right\}
\end{aligned}
$$

where $\theta$ and $\phi$ are the polar and azimuthal angles of the photoelectron. The formulas for nondipole parameters are derived by Bechler and Pratt, Cooper, and Amusia et al. $[14,1,9]$. The expressions for the nondipole parameters $\gamma(\omega)$ and $\delta(\omega)$ involving dipole and quadrupole matrix elements and photoelectron phase shifts in the case of an arbitrary angular momentum $l$ of a bound electron can be found in Refs. $[13,1,3,4,8,9]$.

\section{COMPUTATIONAL PROCEDURE}

In this paper we concentrate on the $3 s$ photoionization of the Ar atom. Determination of the nondipole asymmetry parameter $\gamma_{3 s}(\omega)$ requires accurate evaluation of electric dipole $(E 1)$ and electric quadrupole $(E 2)$ matrix elements between the initial bound states and the final continuum states. In addition, $p$ and $d$ wave phase shifts are to be correctly

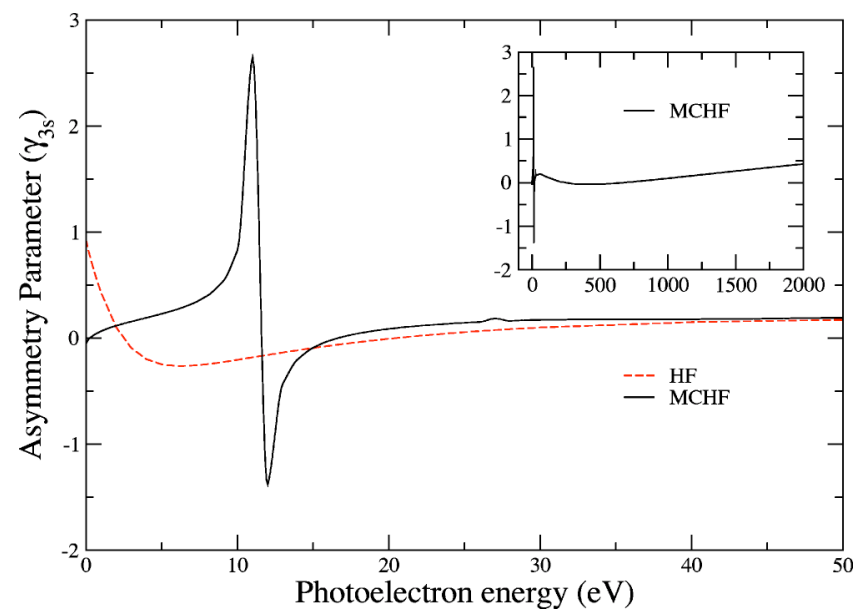

FIG. 4. (Color online) Nondipole asymmetry parameter $\gamma_{3 s}(\omega)$ as a function of photoelectron energy.
TABLE I. Photoelectron energies $(\mathrm{eV})$ at which zeros occur in the parameters.

\begin{tabular}{lcc}
\hline \hline & $\mathrm{HF}$ & $\mathrm{MCHF}$ \\
\hline $\cos \left(\delta_{q}-\delta_{p}\right)$ & $3.0,300$ & $0.3,300$ \\
$\mathrm{DP}$ & & 12.0 \\
$\mathrm{QP}$ & $21.0,700$ & $17.0,700$ \\
$\gamma_{3 s}$ & $3,21,300,700$ & $0.3,12,17,300,700$ \\
\hline \hline
\end{tabular}

evaluated. First of all we calculated numerical HF wave functions for the initial argon $3 s^{2} 3 p^{61} S$ bound state and the final $3 s 3 p^{6} k p^{1} P$ for the dipole matrix elements and $3 s 3 p^{6} k s^{1} S$ and $3 s 3 p^{6} k d^{1} D$ states for the quadrupole matrix elements for photoelectron energies from threshold to $2 \mathrm{keV}$. With these wave functions and the phase shifts of the final continuum states we calculated the dipole and the quadrupole matrix elements. These matrix elements and phases are then used to calculate the nondipole parameter $\gamma_{3 s}(\omega)$. To account for electron correlation and polarization we calculated wave functions for the initial and the final states in the MCHF approximation. The initial bound state of argon is prepared by including all the configurations generated by single and double electron replacements of the outermost $3 s$ and $3 p$ orbitals with the excited $3 d, 4 s$, and $4 p$ orbitals. The excited orbitals are calculated variationally in the selfconsistent procedure. It has been found that the $3 d$ configurations mix very strongly with the ground state of argon. The final continuum states for the dipole and the quadrupole matrix elements are calculated with a number of configurations prepared similarly through replacement of outermost $3 s$ and $3 p$ orbitals with the excited $3 d, 4 s$, and $4 p$ orbitals. These MCHF wave functions along with the phase shifts are then used to calculate the nondipole parameter $\gamma_{3 s}(\omega)$.

\section{RESULTS}

We present the results of the calculation of the nondipole parameter $\gamma_{3 s}(\omega)$ for the $3 s$ subshell ionization of $\mathrm{Ar}$ atom in both the $\mathrm{HF}$ and the MCHF approximations to determine the effect of electron correlation. The results are reported for photoelectron energies from threshold to $2 \mathrm{keV}$. In Fig. 1 we present the dipole matrix elements in the MCHF and HF approximations as a function of photoelectron energy from threshold to $500 \mathrm{eV}$. The dipole matrix elements in the $\mathrm{MCHF}$ approximation change sign near the threshold. But in the HF approximation they do not change sign. Near the threshold there is a large difference between the HF and the $\mathrm{MCHF}$ results but from $100 \mathrm{eV}$ photoelectron energy both results overlap. The quadrupole matrix elements in both the approximations are shown in Fig. 2 as a function of photoelectron energy. Both results change sign near the threshold. There is a difference between the two results near the threshold but above $100 \mathrm{eV}$ the two results overlap.

In Fig. 3, we also present results for $\cos \left(\delta_{q}-\delta_{p}\right)$ in both the HF and the MCHF approximations as a function of photoelectron energy. Each partial-wave phase shift is the sum of 
TABLE II. Comparison of the values of nondipole parameter $\gamma$ with other theoretical results.

\begin{tabular}{|c|c|c|c|c|c|}
\hline \multirow{2}{*}{$\begin{array}{l}\text { Photoeletron } \\
\text { energy }(\mathrm{eV})\end{array}$} & \multicolumn{2}{|c|}{ Present } & \multirow[t]{2}{*}{ Cooper $^{\mathrm{a}}$} & \multirow[t]{2}{*}{$\mathrm{DJC}^{\mathrm{b}}$} & \multirow[t]{2}{*}{$\mathrm{TNY}^{\mathrm{c}}$} \\
\hline & MCHF & $\mathrm{HF}$ & & & \\
\hline 1 & 0.0653 & 0.4288 & & & \\
\hline 2 & 0.1173 & 0.1011 & & & \\
\hline 3 & 0.1566 & -0.0944 & & & \\
\hline 4 & 0.1923 & -0.1990 & & & \\
\hline 5 & 0.2289 & -0.2476 & & & \\
\hline 6 & 0.2705 & -0.2633 & & & \\
\hline 7 & 0.3232 & -0.2601 & & & \\
\hline 8 & 0.3985 & -0.2464 & & & \\
\hline 9 & 0.5268 & -0.2271 & & & \\
\hline 10 & 0.8259 & -0.2050 & & & \\
\hline 12 & -1.3873 & -0.1585 & & & \\
\hline 14 & -0.1980 & -0.1140 & & & \\
\hline 16 & -0.0283 & -0.0735 & & & \\
\hline 18 & 0.0445 & -0.0376 & & & \\
\hline 20 & 0.0869 & -0.0060 & & -0.0104 & \\
\hline 40 & 0.1774 & 0.1507 & & 0.1419 & \\
\hline 60 & 0.1997 & 0.1784 & & 0.1611 & \\
\hline 80 & 0.1719 & 0.1670 & & 0.1495 & \\
\hline 100 & 0.1400 & 0.1431 & 0.14 & 0.1288 & 0.104 \\
\hline 200 & 0.0240 & 0.0264 & 0.03 & 0.0269 & 0.0148 \\
\hline 500 & -0.0339 & -0.0378 & -0.03 & -0.0340 & -0.0296 \\
\hline 1000 & 0.0986 & 0.1004 & 0.10 & 0.1079 & 0.1030 \\
\hline 1500 & 0.2666 & 0.2703 & & 0.2762 & 0.2680 \\
\hline 2000 & 0.4289 & 0.4332 & 0.42 & 0.4339 & 0.4290 \\
\hline
\end{tabular}

${ }^{\mathrm{a}}$ Reference [1].

${ }^{\mathrm{b}}$ Reference [4].

${ }^{\mathrm{c}}$ Reference [7].

Coulomb and residual phase shifts. Notice that there is a change of sign in both results. Although there is a difference between the values of MCHF and HF results near the threshold, they overlap each other at higher photoelectron energy.

The nondipole asymmetry parameter $\gamma_{3 s}(\omega)$ for the $3 s$ subshell ionization is shown in Fig. 4 as a function of photoelectron energy. The onset on this figure represents its behavior at high photoelectron energies. In the HF approximation the parameter $\gamma_{3 s}(\omega)$ changes sign at four energies in the photoelectron energy range $0-2000 \mathrm{eV}$. The large value of $\gamma_{3 s}(\omega)$ at the threshold is due to the large value of quadrupole matrix element and the small value of the dipole matrix element at threshold. The first and the third zeros occur at $k^{2} \approx 3 \mathrm{eV}$ and $k^{2} \approx 300 \mathrm{eV}$ due to $\cos \left(\delta_{q}-\delta_{p}\right)=0$. The second and fourth zeroes at $k^{2}=21.0 \mathrm{eV}$ and $k^{2}=700 \mathrm{eV}$ are due to change of signs of the quadrupole matrix elements. In the MCHF approximation, as mentioned earlier, the electron correlation and polarization effects are taken into account very effectively by including configurations involving $3 d$, $4 s$, and $4 p$ orbitals in both the initial and the final states. As one can see, electron correlation and polarization effects change the behavior of $\gamma_{3 s}(\omega)$ dramatically at low photoelectron energies. The positions of the zeros are shifted to- wards the threshold and one additional zero and a maximum occur near the threshold. The first and the second zero are shifted to $k^{2}=0.3 \mathrm{eV}$ and $k^{2}=17 \mathrm{eV}$, respectively, due to $\cos \left(\delta_{q}-\delta_{p}\right)=0$ and the change of sign of the quadrupole matrix elements. Additional zero occurs at $k^{2}=11.5 \mathrm{eV}$ due to the change of sign of the dipole matrix elements. The fourth and the fifth zeros occur again at $k^{2}=300 \mathrm{eV}$ and $k^{2}$ $\approx 700 \mathrm{eV}$, similar to the HF results. The large maximum at $11 \mathrm{eV}$ and the sharp maximum at $12 \mathrm{eV}$ are due to the sign variation of the dipole matrix elements. The shift of the positions of the zeros and the large maximum and minimum are due to the large correlation and polarization effects of the photoelectron with the core ion. The oscillatory behavior of the asymmetry parameter $\gamma_{3 s}(\omega)$ near the threshold compared to $\mathrm{HF}$ values is due to the large electron correlation effects.

It should be mentioned that the present $\mathrm{HF}$ results for $\gamma_{3 s}(\omega)$ are in excellent agreement with the HF results obtained by Amusia et al. [9]. Near the threshold $0-50 \mathrm{eV}$ the MCHF results, which include electron correlation, agree very well qualitatively with RPAE results calculated by Amusia et al. The difference between the two calculations may be attributed to different methods and different sets of configurations used in the two calculations. At high energies our results agrees well with those obtained by Cooper, Derevianko et al., and Amusia et al. [1,4,9].

In Table I we present the photoelectron energies in $\mathrm{eV}$ at which zeros appear in the dipole matrix elements (DP), the quadrupole matrix elements $(\mathrm{QP})$, and the $\cos \left(\delta_{q}-\delta_{p}\right)$ in both the HF and the MCHF approximations. Table II shows the comparison of $\gamma_{3 s}$ between the present results and the other available theoretical data at a few photoelectron energies. It is seen that there is excellent agreement between the present results and the other theoretical findings.

\section{CONCLUSION}

We investigated the effects of electron correlation and polarization on the nondipole asymmetry parameter of the $3 \mathrm{~s}$ subshell of argon. We performed calculations for the photoelectron energy range from threshold to $2 \mathrm{keV}$ using both the $\mathrm{MCHF}$ and the HF approximations. By comparing the $\mathrm{MCHF}$ and the HF results we found that electron correlation effects change the behavior of the nondipole parameters dramatically near the photoionization threshold. This variation suggests that the dipole approximation alone will not correctly describe the photoelectron angular distribution near the photoionization threshold. Near the photoionization threshold the nondipole parameters show oscillatory behavior as a function of photoelectron energy. These nondipole effects decrease with the increase of photoelectron energy. Finally this investigation will stimulate interest for experimental measurements on $\gamma_{3 s}(\omega)$ close to threshold.

\section{ACKNOWLEDGMENT}

We wish to thank Dr. S. T. Manson for valuable discussions. 
[1] J.W. Cooper, Phys. Rev. A 42, 6942 (1990); 47, 1841 (1993).

[2] E.W.B. Dias, H.S. Chakraborty, P.C. Deshmukh, S.T. Manson, O. Hemmers, P. Glans, D.L. Hansen, H. Wang, S.B. Whitfield, D.W. Lindle, R. Wehlitz, J.C. Levin, I.A. Sellin, and R.C.C. Perera, Phys. Rev. Lett. 78, 4553 (1997).

[3] W.R. Johnson, A. Derevianko, K.T. Cheng, V.K. Dolmatov, and S.T. Manson, Phys. Rev. A 59, 3609 (1999).

[4] A. Derevianko, W.R. Johnson, and K.T. Cheng, At. Data Nucl. Data Tables 73, 153 (1999).

[5] A. Derevianko, O. Hemmers, S. Oblad, P. Glans, H. Wang, S.B. Whitfield, R. Wehlitz, I. A Sellin, W.R. Johnson, and D. W Lindle, Phys. Rev. Lett. 84, 2116 (2000).

[6] W.R. Johnson and K.T. Cheng, Phys. Rev. A 63, 022504 (2001).

[7] M.B. Trzhaskovskaya, V.I. Nefedov, and V.G. Yarzhemsky, At. Data Nucl. Data Tables 77, 97 (2001).

[8] M.Ya. Amusia, A.S. Baltenkov, Z. Felfli, and A.Z. Msezane, Phys. Rev. A 59, R2544 (1999).

[9] M.Ya. Amusia, A.S. Balenkov, L.V. Chernysheva, Z. Felfli, and A.Z. Msezane, Phys. Rev. A 63, 052506 (2001).
[10] B. Krassig, M. Jung, D.S. Gemmell, E.P. Kanter, T. Lebrun, S.H. Southworth, and L. Young, Phys. Rev. Lett. 75, 4736 (1995).

[11] M. Jung, B. Krassig, D.S. Gemmell, E.P. Kanter, T. Lebrun, S.H. Southworth, and L. Young, Phys. Rev. A 54, 2127 (1996).

[12] O. Hemmers, G. Fisher, P. Glans, D.L. Hansen, H. Wang, S.B. Whitfield, R. Wehlitz, J.C. Levin, I.A. Sellin, R.C.C. Perera, E.W.B. Dias, H.S. Chakraborty, P.C. Deshmukh, S.T. Manson, and D.W. Lindle, J. Phys. B 30, L727 (1997).

[13] M.Ya. Amusia, A.S. Baltenkov, A.A. Ginsberg, and S.G. Shapiro, Sov. Phys. JETP 41, 14 (1975); M.Ya. Amusia and N.A. Cherepkov, Case Studies in Atomic Physics (North-Holland, Amsterdam, 1975), Vol. 5, p. 155.

[14] A. Bechler and R.H. Pratt, Phys. Rev. A 39, 1774 (1989); 42, 6400 (1990).

[15] J.H. Scofield, Phys. Rev. A 40, 3054 (1989); Phys. Scr. 41, 59 (1990).

[16] M.O. Krause, Phys. Rev. 177, 151 (1969).

[17] F. Wuilleumier and M.O. Krause, Phys. Rev. A 10, 242 (1974).

[18] H. P. Saha (unpublished). 